\title{
Kompetenzmodell der Modellierkompetenz - Die Rolle abduktiven Schließens beim Modellieren
}

\author{
Dirk Krüger $^{1}$ iD $\cdot$ Annette Upmeier zu Belzen² \\ Eingegangen: 1. Oktober 2020 / Angenommen: 5. Mai 2021 / Online publiziert: 11. Juni 2021 \\ (c) Der/die Autor(en) 2021
}

\section{Zusammenfassung}

In diesem theoretischen Beitrag wird das Kompetenzmodell der Modellkompetenz durch Integration der Theorie des abduktiven Schließens ausdifferenziert und als Kompetenzmodell der Modellierkompetenz präsentiert. Abduktives Schließen in der Biologie heißt, ein biologisches Phänomen durch einen kausalen Rückschluss theoriebasiert oder kreativ bestmöglich zu erklären. Kreativität innoviert im Sinne der Erkenntnisgewinnung durch neues theoretisches Wissen den Prozess des Modellierens. Im ausdifferenzierten Kompetenzmodell wird abduktiv schließendes Erklären als neues Niveau hinzugefügt. Beim abduktiv schließenden Erklären bildet die Herstellung eines Modells die Basis für einen sich möglicherweise anschließenden deduktiven Überprüfungsprozess. Dieser umfasst bei der Anwendung des Modells das Ableiten von Hypothesen aus dem Modell und deren Testung. Bei dieser Anwendung eines Modells als Forschungswerkzeug stehen, im Gegensatz zur Anwendung als verständniserzeugendes Medium, prozedurale sowie epistemische Aspekte des Modellierens im Vordergrund. Fachdidaktische Arbeiten aus verschiedenen Disziplinen weisen auf die Bedeutung des abduktiven Schließens beim Modellieren hin. Ausgehend davon stellt sich die Frage, ob und wie abduktives Schließen beim Modellieren auf der Basis von theoretischem Fachwissen oder kreativer Innovation die Bedeutung eines Modells als Forschungswerkzeug hervorhebt.

Die Berücksichtigung des abduktiven Schließens beim Modellieren stößt Vorhaben in drei Forschungsbereichen an: (1) Bezogen auf Grundlagenforschung gilt es zu klären, inwieweit die erfolgreiche Modellierung eines Phänomens von theoretischen Vorkenntnissen abhängt. (2) Bezogen auf die Erfassung von Modellierkompetenz ist zu prüfen, inwieweit unterschiedliche biologische Kontexte abduktives Schließen anregen. (3) Mit Blick auf eine Förderung von Modellierkompetenz ist zu untersuchen, inwieweit beim Modellieren biologisches Fachwissen für abduktives Schließen bei der Herstellung eines Modells dabei hilft, in eine deduktive Modellanwendung überzuleiten.

Schlüsselwörter Modellierkompetenz $\cdot$ Modellieren $\cdot$ Logisches Schließen $\cdot$ Abduktion

Dirk Krüger

dirk.krueger@fu-berlin.de

1 Institut für Biologie, Fachbereich Biologie, Chemie und Pharmazie, Didaktik der Biologie, Freie Universität Berlin, Schwendenerstraße 1, 14195 Berlin, Deutschland

2 Institut für Biologie, Fachdidaktik und Lehr-/Lernforschung Biologie, Humboldt-Universität zu Berlin, Unter den Linden 6, 10099 Berlin, Deutschland 


\title{
Framework for Modeling Competence-The Role of Abductive Reasoning During Modeling
}

\begin{abstract}
In this theoretical contribution, the framework for model competence is expanded into the framework of modeling competence by integrating the theory of abductive reasoning. Abductive reasoning in biology means to explain a biological phenomenon in the best possible way through a causal inference, either theory-based or creatively. Creativity innovates the process of modeling in the sense of gaining knowledge through new theoretical insight. In the expanded competence model, abductive reasoning is added as a new level. The process of model creation provides a basis for a subsequently deductive verification process, i.e., deriving hypotheses from the model and testing them in the sense of model application. In this application of the model as a research tool, as opposed to its application as an understanding-generating medium, procedural as well as epistemic aspects of modeling are in the foreground. Educational studies in various disciplines point to the importance of abductive reasoning in modeling. Prior research indicates that in modeling, abductive reasoning based on theoretical expertise or creative innovation emphasizes the importance of models as research tools. This also shows the importance of theoretical content knowledge and creativity in modeling.

The consideration of abductive reasoning in modeling initiates three research areas: (1) With regard to basic research, it is important to clarify to what extent successful modeling of a phenomenon depends on prior theoretical knowledge. (2) With regard to the assessment of modeling competence, it should be examined to what extent different biological contexts enable abductive reasoning. (3) With regard to promoting modeling competence, it should be examined to what extent biological content knowledge fosters abductive reasoning to lead over to deductive model application.
\end{abstract}

Keywords Modeling competence $\cdot$ Modeling $\cdot$ Logical reasoning $\cdot$ Abduction

\section{Einleitung}

Das Kompetenzmodell der Modellkompetenz (Upmeier zu Belzen und Krüger 2010; Krüger et al. 2018) hat dazu beigetragen, zwei zentrale Zwecke bei der Anwendung von Modellen theoretisch $\mathrm{zu}$ beschreiben: Modelle mit dem kommunikativen Zweck, aktuelles Wissen als Medium zu repräsentieren sowie Modelle mit dem Zweck, als Forschungswerkzeuge die Generierung neuer Erkenntnisse zu ermöglichen. Modelle als Medien einzusetzen hilft beispielsweise dabei, biologische Strukturen zu beschreiben und deren Funktion oder Beteiligung an Prozessen zu erklären. Modelle als Forschungswerkzeuge einzusetzen bedeutet darüber hinaus, ein Modell zu entwickeln und daraus Voraussagen über zukünftiges Verhalten eines Phänomens unter bestimmten Bedingungen abzuleiten und gegebenenfalls empirisch zu überprüfen, was bestenfalls zu neuen Einsichten und damit zu neuen Erklärungen führt (Krüger et al. 2018).

Darüber hinaus werden beim Prozess des Modellierens zwei Perspektiven unterschieden: Die Herstellung und die Anwendung des Modells (Mahr 2015; Gouvea und Passmore 2017). Ein als Medium eingesetztes Modell wird hergestellt, um es zum Beschreiben und Erklären aktuellen biologischen Fachwissens anzuwenden. Dabei liegt der Schwerpunkt auf der Perspektive der Herstellung eines Modells, nämlich die Realität akkurat im Modell zu repräsentieren, weil es dann in didaktischer Vermittlungsabsicht eingesetzt werden soll (Upmeier zu Belzen und Krüger 2010; Krüger et al. 2018). Demgegenüber wird ein als Forschungswerk- zeug eingesetztes Modell hergestellt, um es zum Ableiten von Hypothesen über bisher nicht erforschte biologische Phänomene anzuwenden. Der Schwerpunkt liegt dabei auf der Perspektive der Anwendung, also aus dem Modell Zusammenhänge zu postulieren, weil durch nachfolgende Untersuchungen neue Erkenntnisse über das unbekannte Phänomen gewonnen werden sollen (Krüger et al. 2018).

Das Kompetenzmodell der Modellkompetenz wird zum Kompetenzmodell der Modellierkompetenz erweitert, indem die erkenntniserweiternde Funktion des Erklärens eines Phänomens bei der Herstellung eines Modells integriert wird (Johnson und Krems 2001; Rocksén 2016; Kampourakis und Niebert 2018). Diese Integration beeinflusst allerdings auch die Perspektive der Anwendung des Modells als Forschungswerkzeug.

Im Kompetenzmodell der Modellkompetenz wurde in der Teilkompetenz Zweck von Modellen das Erklären bisher als mittleres Niveau adressiert. Breiter als im deutschen Sprachverständnis bedeutet Explanation neben dem Erklären für mediale Zwecke auch die anspruchsvolle Suche nach einer in der Vergangenheit liegenden Ursache für ein Phänomen (Johnson und Krems 2001; Rocksén 2016; Kampourakis und Niebert 2018). Bei dieser kognitiven Leistung wird durch abduktives Schließen ein Phänomen bestmöglich erklärt (Johnson und Krems 2001). Bezüglich des Begriffs explanation (Rocksén 2016; Ke und Schwarz 2019) heißt das, dass eine auf didaktische Vermittlungsabsicht reduzierte Betrachtung des Begriffs Erklären in der Bedeutung „deutlich machen“ (Duden 2020) von einer erkennt- 
niserweiternden Funktion des Begriffs Erklären in der Bedeutung „begründen“ (Duden 2020) zu trennen ist.

Mit der Ausdifferenzierung des Erklärens im Kompetenzmodell der Modellierkompetenz bleibt einerseits die didaktische, sachbezogen verständniserzeugende Funktion des Erklärens erhalten, die dem Zweck der medialen Nutzung des Modells dient. Andererseits vermittelt die Integration einer abduktiv schließenden, erkenntniserweiternden Funktion des Erklärens im Kompetenzmodell bereits bei der Herstellung eines Modells eine Untersuchungsabsicht, die eine Anwendung des Modells über deduktiv abgeleitete Hypothesen anstoßen kann. Damit hebt die abduktiv schlieBende Funktion des Erklärens die Bedeutung des Modells als Forschungswerkzeug hervor.

Durch den Begriffswechsel von Modellkompetenz zu Modellierkompetenz wird außerdem das prozedurale und epistemische Potenzial beim Modellieren gegenüber der traditionell vorherrschenden ontologischen Betrachtung von Modellen stärker betont (vgl. Bailer-Jones 1999). Dies erfolgt aufbauend auf grundlegenden theoretischen Arbeiten, die drei Wissensformen beschreiben (Hodson 2014; Kind und Osborne 2017) und hier auf Modelle und das Modellieren bezogen werden (Chiu und Lin 2019): (i) Die ontologische Perspektive beim Arbeiten mit Modellen als
Anschauungsobjekte für fachwissenschaftliches Lernen (learning science). (ii) Die prozedurale Perspektive, wenn beim Modellieren Modelle entwickelt, getestet, verändert und gegebenenfalls gegenständlich repräsentiert werden (doing science). (iii) Die epistemische Perspektive im Sinne von Nature of Science (Heering und Kremer 2018) bei der Arbeit mit Modellen als veränderbare Wissensbestände, die neue Forschungsfragen anstößt (learning about science).

Auch themenverwandte Arbeiten aus anderen Sprachen nutzen die auf den Prozess bezogene Bezeichnung modeling competence (Nicolaou und Constantinou 2014; Chiu und Lin 2019) oder benennen learning progressions mit scientific modeling (Schwarz et al. 2009). Dies legt sprachlich eine stärkere Gewichtung auf prozedurale Anteile beim Modellieren als auf ontologische Anteile des Modells, was die Einführung des Begriffs Modellierkompetenz mit der intendierten Einordnung der beschriebenen Kompetenzen in den Bereich Erkenntnisgewinnung stützt. Dieser begriffliche Schritt wurde für den englischen Sprachraum bereits im framework for modeling competence vollzogen (Upmeier zu Belzen et al. 2019).

Um die Bedeutung des abduktiven Schließens für das Modellieren hervorzuheben, werden im Beitrag die Kom-

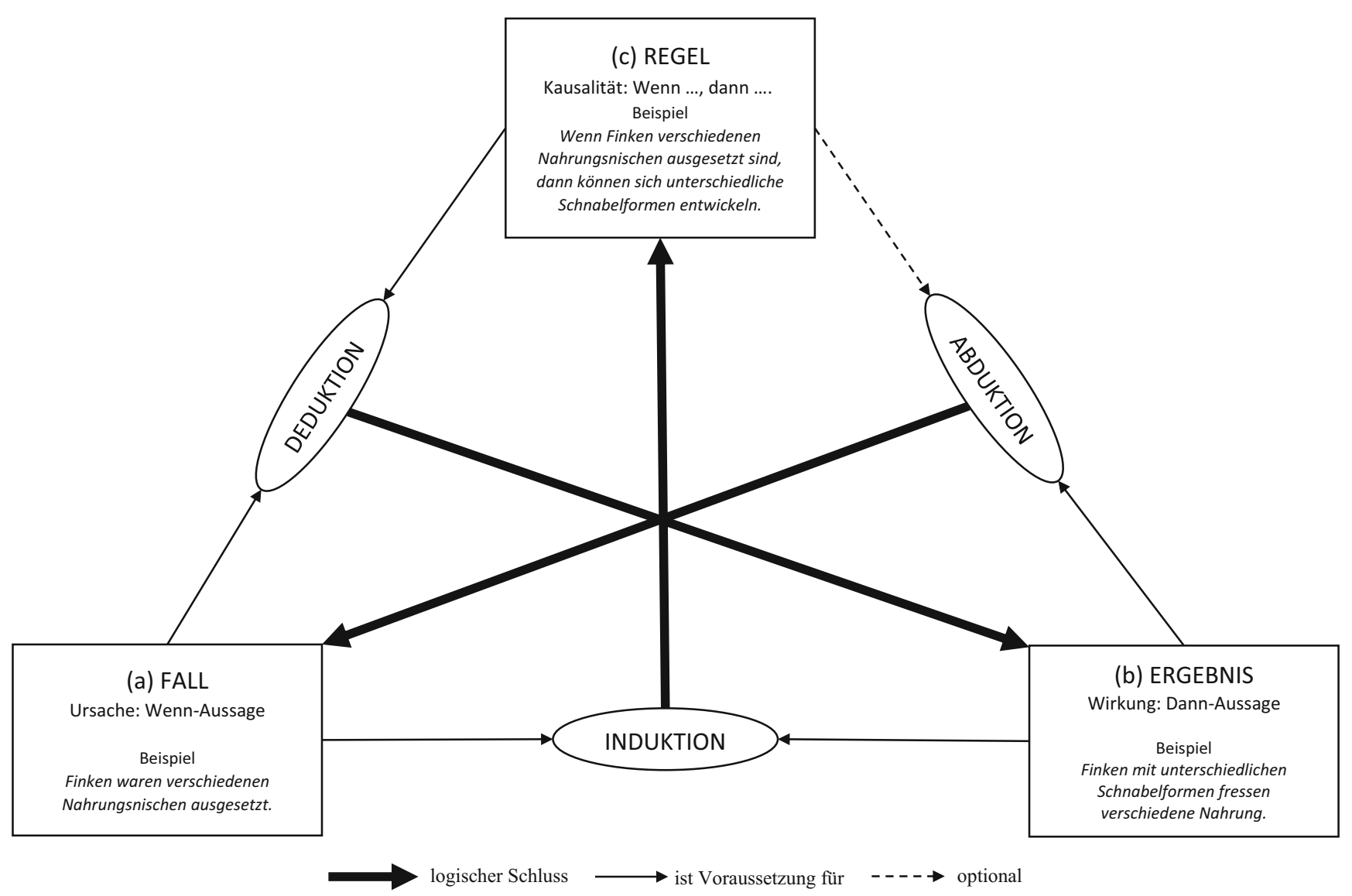

Abb. 1 Beziehungen zwischen Deduktion, Induktion und Abduktion (verändert nach Sturm 2006) sowie Illustration durch ein Beispiel 
ponenten der Theorie des abduktiven Schließens (Johnson und Krems 2001) vorgestellt und in den zyklischen Prozess des Modellierens integriert. Dies macht eine Klärung der Begriffe Modell, Herstellungs- und Anwendungsperspektive notwendig (Mahr 2015). Die theoretischen Überlegungen zum abduktiven Schließen beim Modellieren werden durch empirische Arbeiten aus den Bereichen Mathematik (Park und Lee 2018) und Geografie (Clement 2008; Oh 2011, 2019) gestützt.

Das Einbeziehen des abduktiv schließenden Erklärens in das Kompetenzmodell der Modellierkompetenz führt zu fachdidaktischem Forschungsbedarf in drei Bereichen: Grundlagenforschung, Kompetenzerfassung und Kompetenzförderung. In der Grundlagenforschung wird es darum gehen, die Bedeutung abduktiven Schließens bei der Modellherstellung für den Übergang in die forschende Anwendungsperspektive beim Modellieren zu belegen. Im Sinne der Kompetenzerfassung muss geprüft werden, ob sich Kompetenzen in problemhaltigen Aufgabenstellungen mit Modellen vermehrt zeigen, wenn diese aussagekräftige abduktive Schlüsse ermöglichen. Schließlich gilt es unter einer Förderperspektive zu untersuchen, wann und wie Lernende von einem abduktiven Schließen zur deduktiven Prüfung des Modells übergehen.

\section{Logisches Schließen und biologisches Erklären}

Es werden drei Arten logischen Schließens unterschieden, die in verschiedenen Phasen des Modellierens besondere Bedeutung erlangen: Deduktion, Induktion und Abduktion (Peirce 1978; Magnani 1999, 2004; Mahootian und Eastman 2009). Bei der Deduktion wird aus einer allgemeinen Regel (Kausalität, Abb. 1 (c)) und einem Einzel-Fall (Ursache, Abb. 1 (a)) ein Ergebnis (Wirkung, Abb. 1 (b)) postuliert. Sofern die Regel wahr ist, muss jeder Fall gemäß der Regel zu einem bestimmten Ergebnis führen. Deduktive Schlüsse sind aufgrund der vorhandenen Regel wahrheitserhaltend und logisch einwandfrei, sind jedoch durch die Ableitung einer Regel auf Einzelfälle nicht erkenntniserweiternd (Meyer 2009). Bei der Induktion wird aus kausal interpretierten Einzelbeobachtungen eines Falles (Abb. 1 (a)) und eines Ergebnisses (Abb. 1 (b)) eine verallgemeinernde Regel entwickelt (Abb. 1 (c)). Induktive Schlüsse sind damit gehaltserweiternd, aber logisch nicht einwandfrei. Sie entgrenzen beim Generalisieren lediglich den Geltungsbereich an Einzelbeobachtungen bereits identifizierter Zusammenhänge (Peirce 1978; Reichertz 2013). Bei der Abduktion wird für ein beobachtetes Ergebnis (Abb. 1 (b)) und möglicherweise auf der Basis bestehender Regeln (Abb. 1 (c)) eine Ursache als beste Erklärung generiert (Abb. 1 (a); inference to the best explanation,
Harman 1965; educated guess, Clement 2008). Wenn dabei kreative Ideen bisherige Erklärungen innovieren, ist abduktives Schließen gehalts- und erkenntniserweiternd (Habermas 1968; Wirth 2003; Schurz 2008; Meyer 2009). Das Verhältnis der drei Arten logischen Schließens wird in folgendem Zitat zusammengefasst: „Deduction proves that something must be; Induction shows that something actually is operative; Abduction merely suggests that something may be" (Hervorhebungen bei Peirce 1978, CP 5.171). Die Abduktion ist durch die rückwärts gerichtete Argumentation geeignet, Phänomene retrodiktiv zu lösen (Magnani 2004; Oh 2019), sie kann also Aussagen über den kausalen Zusammenhang von in der Vergangenheit liegenden Ereignissen treffen. Die Beobachtung der Darwinfinken mit verschiedenen Schnabelformen und unterschiedlichem Nahrungsspektrum (Ergebnis; Abb. 1 (b); Grant und Weiner 1999) kann unter Rückgriff auf die Reaktionsnorm (Regel; Abb. 1 (c)) zur Erklärung genutzt werden, um darauf zu schließen, dass ursprüngliche Finken mit einer Schnabelform in einem Lebensraum mit vielfältigem Nahrungsangebot gelebt haben (Fall; Abb. 1 (a)) und sich die Artenvielfalt durch Mutation und Selektion entwickelt hat. Alternative Erklärungen könnten sein, dass die Finkenvielfalt schon immer vorhanden war.

In der Biologie werden spezifische Formen des Erklärens genutzt: Funktionale Erklärungen helfen in der Morphologie, Physiologie und Molekularbiologie, kausalanalytisch mit Bezug auf Komponenten und Strukturen von Prozessen zielgerichtet und damit teleonomisch zu antwor-

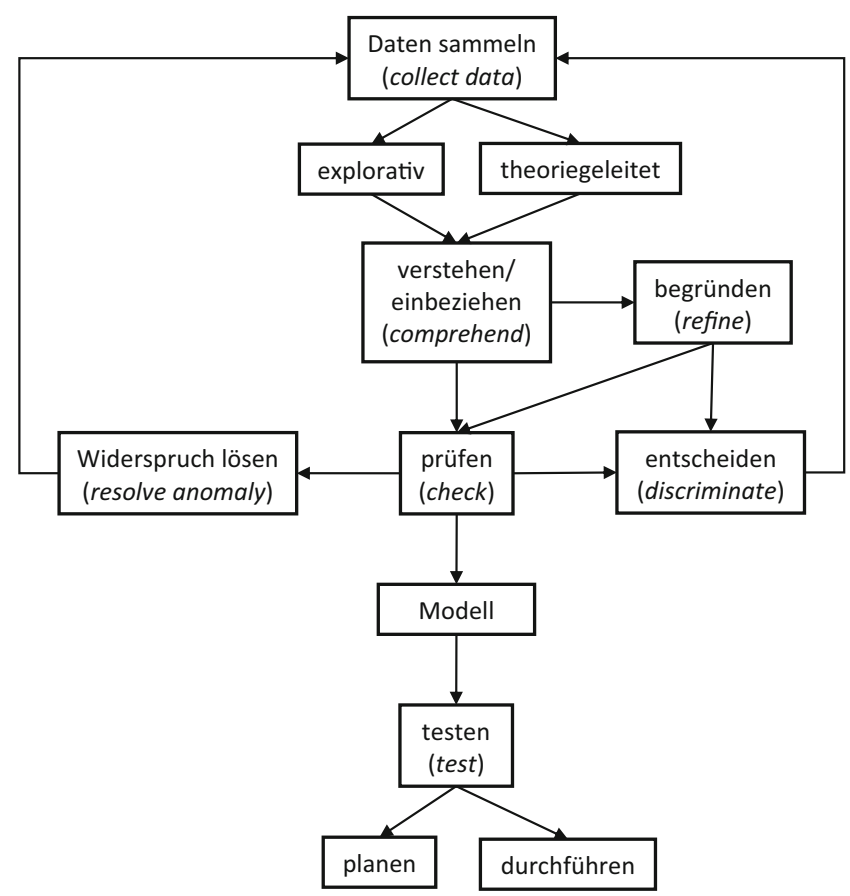

Abb. 2 Idealtypischer Prozess mit Komponenten des abduktiven Schließens (verändert nach Johnson und Krems 2001) 
ten (Kampourakis und Niebert 2018). Dies ist mit der Wozu ist etwas entstanden-Frage verbunden. Proximate Erklärungen liefern im Sinne von Nahursachen Antworten auf die Wie kommt etwas zustande-Frage, zum Beispiel auf physiologischer Ebene, während ultimate Erklärungen in der Evolutionsbiologie im Sinne von Fernursachen versuchen, die Wie ist etwas zustande gekommen-Frage $\mathrm{zu}$ beantworten. Davon unterschieden werden biologisch nicht adäquate Erklärungen, die intentional und damit teleologisch im Sinne von Zielursachen auf die Welches Ziel wird verfolgtFrage eher aus einer menschlichen Perspektive antworten (Langlet 2016; Kampourakis und Niebert 2018; Trommler und Hammann 2020). Biologische Erklärungen kommen durch abduktives Schließen maßgeblich in der Evolutionstheorie zustande (Kind und Osborne 2017), wenn Phänomene beobachtet werden, theoretische Überlegungen herangezogen werden, Kreativität einbezogen wird und unsichere Erklärungen über das Beobachtete entwickelt werden (Johnson und Krems 2001).

Je nach individueller Vorkenntnis kann in einen abduktiven Schluss mehr oder weniger theoretisches Wissen einfließen. Habermas (1968, S. 140) unterscheidet zwischen explanatorischer und innovatorischer Abduktion, was nach Schurz (2008) als selective und creative abduction benannt wird, worunter sich nach Bonfantini und Proni (1985; zitiert nach Wirth 2003) drei Formen des abduktiven Schließens fassen lassen: Die selektive Abduktion mit der überkodierten Abduktion als Erklärung auf der Basis einer vorausgesetzten Theorie und der unterkodierten Abduktion, bei der aus vielen nach der am besten passenden theoretischen Grundlage gesucht wird sowie die innovatorische Abduktion. Die innovatorische Abduktion ist die kreative Abduktion, bei der ein Phänomen mit einer neu erfundenen Regel - im Sinne einer theoretischen Grundlage - erklärt wird. Nur die innovatorische (kreative) Abduktion bringt neue Ideen in die wissenschaftliche Diskussion ein und ist somit erkenntniserweiternd (Wirth 2003; Meyer 2009; Reichertz 2013).

\section{Theorie des abduktiven Schließens}

Johnson et al. (1994) sowie Krems und Johnson (1995) schlagen eine kognitionspsychologische Theorie abduktiven Schließens mit sieben Komponenten vor. Diese Theorie des abduktiven Schließens (Johnson und Krems 2001) beschreibt einen kontinuierlichen, impliziten Prozess, der nicht in einer festgelegten Reihenfolge durchlaufen werden muss (Abb. 2; Krems et al. 1997) und zu einem konsistenten und von Redundanzen befreiten Erklärungsmuster führen kann. Idealtypisch beginnt der Prozess des abduktiven Schließens nach Johnson und Krems (2001) mit der Wahrnehmung eines Phänomens, zu dem Daten explo- rativ oder theoriegeleitet gesammelt werden (collect data, Abb. 2). Anschließend werden diese Daten in einem subjektiven Prozess der Hypothesenbildung in ein bestehendes gedankliches Modell einbezogen und vorläufig verstanden (comprehend, Abb. 2). Dieser domänenabhängige Prozess geschieht zunächst lokal, also auf der Basis einzelner Daten und unabhängig von bereits vorhandenen anderen Daten. Liegt nur eine mögliche Erklärung für das Phänomen vor (überkodierte oder kreative Abduktion), wird geprüft (check, Abb. 2), ob neue Daten im Widerspruch zum bisherigen Modell stehen oder in Bezug auf dieses unverständlich bleiben (resolve anomaly, Abb. 2). Tritt dies ein, werden weitere Daten gesammelt (collect data). Liegen mehrere mögliche Erklärungen vor (unterkodierte oder kreative Abduktion), werden alternative potenziell plausible Erklärungen bewertet (refine, Abb. 2), was es notwendig macht (discriminate, Abb. 2), eine aus den potenziell plausiblen Erklärungen auszuwählen. Bei der Prüfung auf Konsistenz (check) werden auch unwahrscheinliche Erklärungen einbezogen. Um eine Entscheidung treffen zu können, kann dies zum Sammeln weiterer Daten führen (collect data). Gelingt die Prüfung auf Konsistenz und Widerspruchsfreiheit nicht (check), werden weitere potenziell plausible Erklärungen bewertet (discriminate). Obwohl es im Modell bei Johnson und Krems (2001) mit test (Abb. 2) darum geht, diese Unsicherheit über unwahrscheinliche Erklärungen auszuräumen, lässt sich dieser Denkschritt auf ein abduktiv entwickeltes Modell ausweiten, aus dem deduktiv Hypothesen abgeleitet werden, die zu überprüfen sind (Magnani 1999; ,abductive model evaluation“, Clement 2008, S. 483).

Die im idealtypischen Prozess beschriebenen Komponenten verdeutlichen, dass abduktives Schließen nach einer Phase der Exploration unter Bezug auf theoretisches Wissen in ein kreatives Entscheiden, Begründen und Prüfen überleitet, das anspruchsvoll ist und in einer konsistenten Erklärung münden kann (Clement 2008).

\section{Modellieren als Herstellen und Anwenden eines Modells}

Um im Folgenden die Bedeutung und den Einfluss des abduktiven Schließens beim Modellieren deutlich zu machen, werden zunächst das hier genutzte Verständnis über Modelle und die damit zusammenhängenden Prozesse ihrer Herstellung und Anwendung erläutert. Dabei entzieht sich der Prozess des Modellierens grundsätzlich einer strengen prozeduralen Beschreibung und Festlegung bestimmter Regeln (Morrison und Morgan 1999). Das liegt auch daran, dass Erfahrungen, Vorstellungen und Theorien des modellierenden Subjekts (Clement 2008) aufgrund der kreativen, innovierenden und subjektiven Erwägungen, die dabei herangezogen werden, den Prozess beeinflussen. Dennoch lassen sich 
beim Modellieren wiederkehrende Elemente identifizieren, die idealtypisch angeordnet einer hypothetisch-deduktiven Forschungslogik folgen (Popper 2005; Langlet 2016). Im Folgenden dient das bei Krell et al. (2016) und Krell et al. (2019) beschriebene Prozessschema des Modellierens als Grundlage für die Integration des abduktiven Schließens.

Wegen der Vielgestaltigkeit von Modellen und da alles Modell werden kann, was von einem Subjekt als Modell aufgefasst wird (Harré 1970; Mahr 2015), findet man keine Eigenschaft, die Modelle objektbezogen ontologisch charakterisiert (Mittelstraß 2005; Mahr 2008). Der Begriff Modell hat so viele Bedeutungen, dass Versuche, diese in einer Definition zu bündeln, methodisch unbrauchbar sind (Mahr 2009). Vielmehr lohnt es, auf epistemischer Ebene zu beschreiben, wie Modelle genutzt werden, um ein Phänomen in der Erfahrungswelt zu verstehen (Bailer-Jones 1999; Krüger et al. 2018; Upmeier zu Belzen et al. 2019). Mahr (2015) beschreibt in seinem Modell des Modellseins, dass ein Subjekt ein Urteil darüber fällt, für welchen Zeitraum etwas eine Identität als Modell erhält und es somit ein Modell sein lässt. Zudem ist die Unterscheidung zwi- schen einem gedachten Modell und seiner Repräsentation, dem im weitesten Sinne gegenständlichen Modellobjekt, relevant für den Modellbegriff nach Mahr (2008). Modelle sind somit als Abstraktionen und Modellobjekte als idealisierte Repräsentationen zu verstehen, bei denen das für das Erkenntnisinteresse Unwesentliche weggelassen wird und verallgemeinernd ausgewählte Bestandteile eines natürlichen Systems als Gemeinsamkeiten einzelner Phänomene herausgearbeitet werden (Godfrey-Smith 2006; Shemwell und Capps 2019). Abstraktion ist damit eine zweckgerichtete Neuschöpfung, die die bestehende Erkenntnis im Sinne von Theorie über die begrenzte Menge der vorliegenden Fälle hinaus erweitert. Das Verhältnis von Modell und Theorie reicht dabei in der wissenschaftstheoretischen Literatur von einer Perspektive von Modellen lediglich als Hilfsmittel, um Theorien in Ausschnitten zu repräsentieren (syntactic view), hin zu einer Perspektive, bei der Theorien als Familien von Modellen aufgefasst werden (semantic view). Letzteres stärkt die Auffassung, dass Modelle selbstständige, bedeutungstragende theoretische Konstrukte sind (Aduriz-Bravo 2019).
Abb. 3 Prozess des Modellierens. In der Herstellungsperspektive (obere Hälfte) wird ein Phänomen (Erfahrungswelt; linke Seite) als Modell (Modellwelt; rechte Seite) durch induktives oder abduktives Schließen konstruiert. In der Anwendungsperspektive (untere Hälfte) wird ausgehend von deduktiv geschlossenen Hypothesen (Modellwelt) das Phänomen empirisch untersucht (Erfahrungswelt) und damit Evidenz für die entwickelte Interpretation des Phänomens erzeugt (verändert nach Krell et al. 2016; Upmeier zu Belzen et al. 2019)

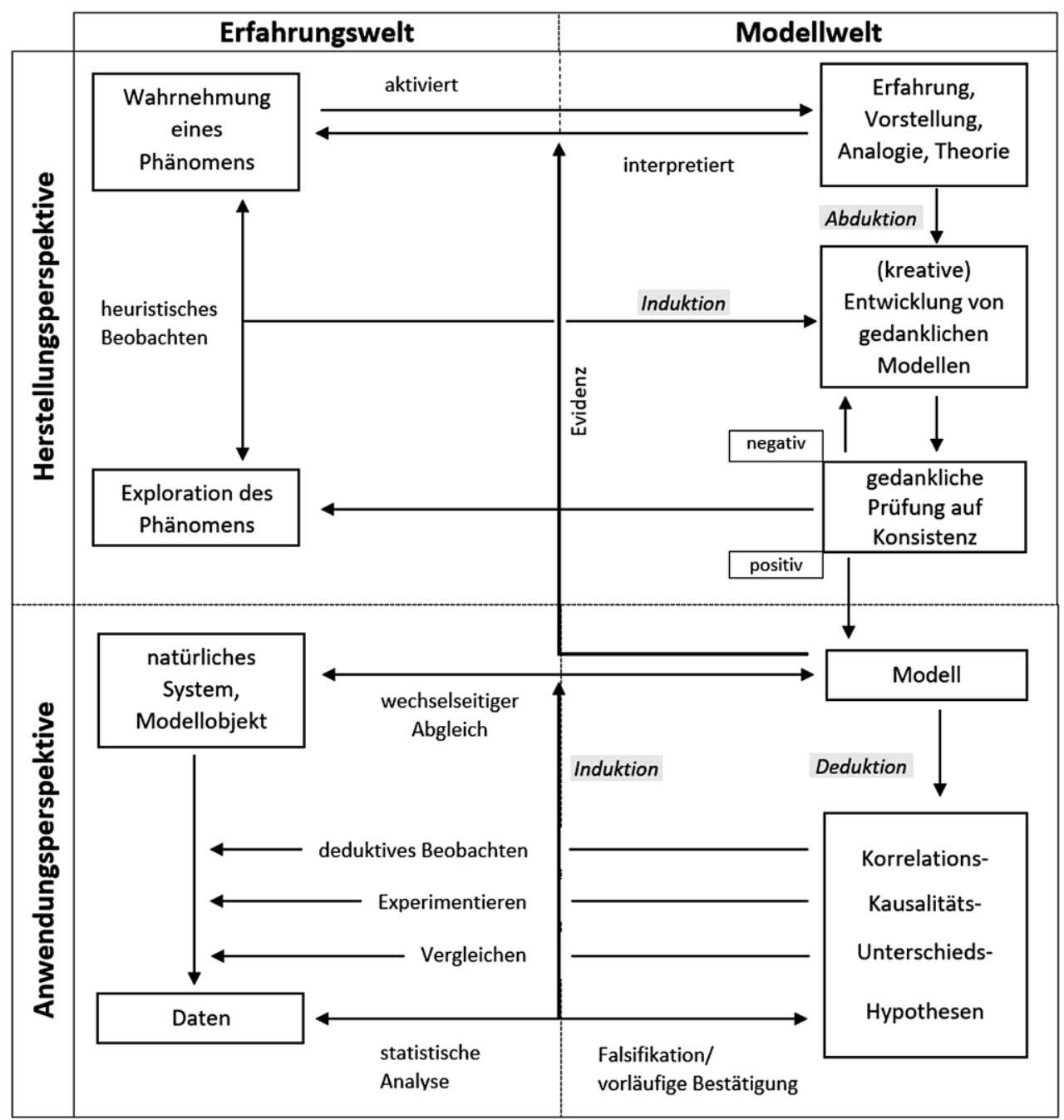


Das Modell bzw. Modellobjekt steht dabei in zwei Beziehungen zu etwas: In der Herstellungsperspektive steht es in der Beziehung zu etwas, von dem es ein Modell ist, und in der Anwendungsperspektive in der Beziehung zu etwas, für das es als Modell eingesetzt wird (Mahr 2009, 2015; Gouvea und Passmore 2017). Diese beiden Beziehungen sind konstitutive und inhärente Aspekte des Modellbegriffs (Passmore et al. 2014; Mahr 2015). Durch sie wird deutlich, dass sich das Modellieren auf eine zweckgerichtete Aktivität eines Subjekts in einem Herstellungs- und einem Anwendungsprozess von Modellen bezieht (Giere et al. 2006; Mahr 2015). Damit werden beim Modellieren prozedurale und epistemische Begründungszusammenhänge relevant (Kind und Osborne 2017), die auch selektive und innovatorische Abduktionen einbeziehen. Die beim abduktiven Schließen durchlaufenen Schritte (Abb. 2) vollziehen sich in der Herstellungsperspektive im Wechsel zwischen dem explorierten Phänomen und dem in Beziehung Setzen von Erfahrungen, Vorstellungen, Analogien und theoretischen Überlegungen bei der kreativen Entwicklung eines gedanklichen Modells und seiner gedanklichen Konsistenzprüfung (Abb. 3).

Ein Modellierungsprozess kann mit der Wahrnehmung eines Phänomens durch heuristische Beobachtung beginnen (Abb. 3; Greve und Wentura 1997). Heuristisches Beobachten erfolgt aus einer Haltung, unvoreingenommen, möglichst vollständig und ohne explizite Vermutungen und Theorien, das Phänomen zu explorieren (Abb. 3), Zusammenhänge zu entdecken oder bestimmte Ausschnitte des Geschehens systematisch zu erfassen und zu ordnen (Greve und Wentura 1997; collect data, comprehend, Abb. 2). Mit heuristischem Beobachten in der Erfahrungswelt werden Hypothesen über Zusammenhänge und möglicherweise
Theorien generiert. Diese können aus Einzelbeobachtungen (Umwelt ändert sich, neue Phänotypen tauchen vermehrt auf, vgl. Birkenspanner bei Baalmann und Kattmann 2000) durch induktives Schließen auf ein verallgemeinerndes Modell (wenn sich die Umwelt ändert, ändert sich eine Population) entstehen, wobei sie vor einer empirischen Testung auf Konsistenz und Widerspruchsfreiheit gegenüber anderen Theorien (Regeln) geprüft werden (Abb. 3). Alternativ wird durch abduktives Schließen (Johnson und Krems 2001; neue Phänotypen tauchen vermehrt auf, vgl. Birkenspanner bei Baalmann und Kattmann 2000) das Phänomen zum Beispiel mit Hilfe von Analogien (wenn etwas dem Untergrund ähnelt, wird es schlecht erkannt, die Umwelt hat sich verändert) erklärt und ebenfalls auf Konsistenz geprüft (Abb. 3; refine, discriminate, check, resolve anomaly, Abb. 2). Führen induktive oder abduktive Schlüsse zu Modellen, lassen sich beim Übergang von der Herstellungs- in die Anwendungsperspektive deduktiv Hypothesen darüber ableiten, wie sich das modellierte Beziehungsgefüge unter bestimmten Bedingungen verhält (Abb. 3; test, Abb. 2). Je nach Art der Hypothese führt dies in unterschiedliche methodische Umsetzungen und damit in entsprechende Arbeitsweisen (Abb. 3; Upmeier zu Belzen und Krüger 2019a). Während Unterschiedshypothesen zum Vergleichen von Strukturen, Gruppen oder Systemen führen, werden Kausalhypothesen durch Experimentieren und Zusammenhangshypothesen durch deduktives Beobachten (Greve und Wentura 1997) untersucht (Abb. 3). Die statistisch analysierten Daten aus dem natürlichen System oder Modellobjekt, das bei fehlendem Zugriff auf das natürliche System eingesetzt werden kann, erlauben es, die Hypothesen zu stützen oder zu falsifizieren (Abb. 3). Kommen Störquellen bei der Datenerfassung als Begründungen

Tab. 1 Kompetenzmodell der Modellierkompetenz (überarbeitet; Krüger et al. 2018)

\begin{tabular}{|c|c|c|c|c|}
\hline Teilkompetenzen & $\begin{array}{l}\text { Niveau I } \\
\text { Exklusive Betrachtung } \\
\text { des Modellobjekts }\end{array}$ & $\begin{array}{l}\text { Niveau II } \\
\text { Sachbezogen verständnis- } \\
\text { erzeugende Erklärung des } \\
\text { Phänomens }\end{array}$ & $\begin{array}{l}\text { Niveau IIIA }^{\mathrm{a}} \\
\text { Abduktiv schließende Erklä- } \\
\text { rung des Phänomens }\end{array}$ & $\begin{array}{l}\text { Niveau IIIB } \\
\text { Hypothetisch-deduktive Unter- } \\
\text { suchung des Phänomens }\end{array}$ \\
\hline $\begin{array}{l}\text { Eigenschaften } \\
\text { von Modellen }\end{array}$ & $\begin{array}{l}\text { Entspricht möglichst } \\
\text { vollständig dem Phäno- } \\
\text { men }\end{array}$ & $\begin{array}{l}\text { Repräsentiert verständlich } \\
\text { und abstrahiert das Phäno- } \\
\text { men }\end{array}$ & $\begin{array}{l}\text { Repräsentiert beste Erklä- } \\
\text { rung des Phänomens }\end{array}$ & $\begin{array}{l}\text { Repräsentiert eine theoretische } \\
\text { Rekonstruktion des Phänomens }\end{array}$ \\
\hline $\begin{array}{l}\text { Alternative } \\
\text { Modelle }\end{array}$ & $\begin{array}{l}\text { Existieren wegen unter- } \\
\text { schiedlicher Objektmerk- } \\
\text { male }\end{array}$ & $\begin{array}{l}\text { Existieren wegen der Kom- } \\
\text { plexität des Phänomens }\end{array}$ & $\begin{array}{l}\text { Existieren wegen unter- } \\
\text { schiedlicher theoretischer } \\
\text { Erklärungen }\end{array}$ & $\begin{array}{l}\text { Existieren wegen konkurrieren- } \\
\text { der theoretischer Vorstellungen }\end{array}$ \\
\hline $\begin{array}{l}\text { Zweck des } \\
\text { Modellierens }\end{array}$ & $\begin{array}{l}\text { Dient dem Beschreiben } \\
\text { und Veranschaulichen des } \\
\text { Phänomens }\end{array}$ & $\begin{array}{l}\text { Dient dem verständlichen } \\
\text { Erklären des Phänomens }\end{array}$ & $\begin{array}{l}\text { Liefert die beste theoreti- } \\
\text { sche Erklärung des Phäno- } \\
\text { mens }\end{array}$ & $\begin{array}{l}\text { Erlaubt mit Hypothesen das } \\
\text { Voraussagen empirischer Er- } \\
\text { gebnisse zum Phänomen }\end{array}$ \\
\hline $\begin{array}{l}\text { Testen von } \\
\text { Modellen }\end{array}$ & $\begin{array}{l}\text { Erfolgt durch Untersu- } \\
\text { chen der Robustheit oder } \\
\text { Funktionalität }\end{array}$ & $\begin{array}{l}\text { Erfolgt durch Überprü- } \\
\text { fen der Passung zwischen } \\
\text { Phänomen und Modell }\end{array}$ & $\begin{array}{l}\text { Erfolgt durch theoretisches } \\
\text { Überprüfen der Erklärung } \\
\text { auf Konsistenz }\end{array}$ & $\begin{array}{l}\text { Erfolgt durch empirisches } \\
\text { Überprüfen von Hypothesen in } \\
\text { der Erfahrungswelt }\end{array}$ \\
\hline $\begin{array}{l}\text { Ändern von } \\
\text { Modellen }\end{array}$ & $\begin{array}{l}\text { Führt zum Beheben von } \\
\text { Mängeln }\end{array}$ & $\begin{array}{l}\text { Führt zum Revidieren bei } \\
\text { fehlender Passung durch } \\
\text { neue Erkenntnisse }\end{array}$ & $\begin{array}{l}\text { Führt zum Revidieren der } \\
\text { Erklärungen bei fehlender } \\
\text { Konsistenz }\end{array}$ & $\begin{array}{l}\text { Führt zum Integrieren von } \\
\text { neuen Erkenntnissen aus falsifi- } \\
\text { zierten Hypothesen }\end{array}$ \\
\hline
\end{tabular}

aTheoretische Erklärungen ergeben sich selektiv aus theoretischen Bezügen oder innovatorisch aus kreativen Ideen 
für die fehlende Passung zwischen den aus dem Modell abgeleiteten Hypothesen und dem untersuchten Phänomen nicht infrage, werden das Modell, das Modellobjekt und damit die Vorstellung über das modellierte Phänomen verändert. Dabei ermöglichen neue Beobachtungen in der Erfahrungswelt induktives Schließen, womit der Prozess der Entwicklung und Testung eines veränderten Modells erneut beginnen kann (Abb. 3; Giere et al. 2006). Auf diese Weise hilft das weiterentwickelte Modell, Evidenz über das Phänomen zu gewinnen (Abb. 3). Indem Modelle auf diese Art Erkenntnisprozesse einleiten, werden sie zu flexiblen intellektuellen Instrumenten naturwissenschaftlicher Erkenntnisgewinnung (Bailer-Jones 1999; epistemic tools, Knuuttila 2011). Diese Funktion geht über das Präsentieren eines Modellobjekts als Kommunikationsmittel hinaus.

\section{Kompetenzmodell der Modellierkompetenz}

Das Kompetenzmodell der Modellierkompetenz (Tab. 1) erweitert das Kompetenzmodell der Modellkompetenz, welches aus fünf Teilkompetenzen und drei Niveaustufen besteht (vgl. Tab. 1; Upmeier zu Belzen und Krüger 2010; Krüger et al. 2018). Das ausdifferenzierte Kompetenzmodell enthält als neues Niveau IIIA das abduktiv schließende Erklären (Tab. 1). Dieses grenzt sich von dem sachbezogen verständniserzeugenden Erklären mit Modellen $\mathrm{ab}$, das im Kompetenzmodell der Modellkompetenz das Niveau II beschreibt (Tab. 1; Upmeier zu Belzen und Krüger 2010). Dabei dient das verständniserzeugende Erklären der Erläuterung eines grundsätzlich gut erforschten Sachverhaltes oder Vorgangs (vgl. Rocksén 2016). Die erkenntniserweiternde Funktion des Erklärens befindet sich nun mit dem abduktiven Schließen als Niveau IIIA im Kompetenzmodell der Modellierkompetenz. Das abduktiv schließende Erklären (Niveau IIIA; Tab. 1) bezieht bei der Herstellung des Modells wie das vorausschauende Prognostizieren (Niveau IIIB; Tab. 1) bei der Anwendung des Modells theoretische oder kreative Erwägungen ein und kann somit bereits im Herstellungsprozess zu neuer Erkenntnis führen. Die abduktiv schließende Erklärung im Niveau IIIA ist wissenschaftlich anspruchsvoll (Johnson und Krems 2001) und läuft, idealtypisch betrachtet, dem Niveau IIIB im Sinne des hypothetisch-deduktiven Erkenntniswegs (Langlet 2016) voraus (Tab. 1).

Der Einbezug einer allgemeinen Theorie abduktiven Schließens beim Modellieren (Johnson et al. 1994; Krems und Johnson 1995), in der in einem komplexen und kreativen Verstehensprozess sequenziell ein Modell hergestellt wird, aus dem kausale Schlüsse gezogen werden, führt ausgehend von der Definition von Modellkompetenz zu einer angepassten Definition der kognitiven Facette von
Modellierkompetenz (vgl. Upmeier zu Belzen und Krüger 2010):

Modellierkompetenz umfasst die Fähigkeiten, beim Herstellen von Modellen einen theoriegeleiteten oder kreativen Erkenntnisprozess zu initiieren, bei der Anwendung von Modellen zweckbezogen Erkenntnisse zu gewinnen, über Modelle mit Bezug auf ihren Zweck zu urteilen und über den Prozess der Erkenntnisgewinnung durch Modelle und das Modellieren zu reflektieren

Die Beschreibungen in den Zellen wurden angepasst, ohne dabei die Inhalte der Niveaus I, II und IIIB gegenüber früheren Beschreibungen zu ändern (Upmeier zu Belzen und Krüger 2010; Krüger et al. 2018). Dabei geht es mit Blick auf die Einordnung in den Kompetenzbereich Erkenntnisgewinnung darum, das Modellieren stärker als das Modell anzusprechen und im Sinne von Kompetenzbeschreibungen aktive Formulierungen zu verwenden (KMK 2020). Die Kompetenzbeschreibungen in den Niveaus I, II und IIIB greifen auf theoretische Ausarbeitungen (Upmeier zu Belzen und Krüger 2010; Krüger et al. 2018) und umfangreiche empirische Arbeiten zurück, die es erlauben, die dargestellte Strukturierung für die Erfassung und Förderung von Modellierkompetenz zu nutzen (u.a. Krüger und Krell 2020; Upmeier zu Belzen und Krüger 2019b; Krell et al. 2016).

Die Niveaus I und II beschreiben das Modellieren in einer medialen Anwendung. Der Fokus liegt auf dem akkuraten Herstellungsprozess eines Modells, damit es der Kommunikation oder dem Aufbau einer fachwissenschaftlich orientierten Kompetenz dient. Die Niveaus IIIA und IIIB beschreiben das Modellieren im Forschungsprozess, der im Herstellen und Anwenden eines Forschungswerkzeuges besteht. Im Niveau IIIB wird durch den hypothetischdeduktiven Einsatz eines Modells zur empirischen Überprüfung seiner Aussagekraft auf den Anwendungsprozess fokussiert.

Das Niveau IIIA wird hier erstmals beschrieben und stellt die zentrale Erweiterung im Kompetenzmodell der Modellierkompetenz dar. Es beschreibt die Fähigkeit, im Herstellungsprozess ein Modell zu entwickeln, das bestmögliche Erklärungen für neue Phänomene liefert, welche konsistent und widerspruchsfrei zu bisherigen Theorien sind. Modellieren ist damit bereits in der Herstellung ein theoretischer oder kreativer Prozess, der, mit Unsicherheit verbunden, das Wissen über ein Phänomen repräsentiert und neue Möglichkeiten der Erklärung bieten kann.

Ausgangspunkt für die Integration von Niveau IIIA war die Ausdifferenzierung des Erklärens in der Teilkompetenz Zweck des Modellierens, die auch im Begriffswechsel zur Modellierkompetenz deutlich wird. Die Integration von Niveau IIIA zeigt sich in allen Teilkompetenzen: In der Teil- 
kompetenz Eigenschaften von Modellen wird erkannt, dass ein Modell eine aktuell beste Erklärung liefert, in der Teilkompetenz alternative Modelle, dass das kreative Spektrum von Modellierenden Ausgangpunkt der Vielfalt ist, in der Teilkompetenz Testen von Modellen gilt, das gedankliche Prüfen der Erklärung auf Konsistenz zu erkennen und beim Ändern von Modellen, dass das gedankliche kritische Abwägen zu Weiterentwicklungen führen kann.

\section{Empirische Einblicke}

Es liegen mittlerweile fachdidaktische Ansätze vor, die zwar das abduktive Schließen mit dem Modellieren verbinden, dabei jedoch die Beziehung zwischen Abduktion und Deduktion nicht vertiefen (Clement und Núñez-Oviedo 2003; Clement 2008; Oh 2019). Die Geowissenschaften treffen wie die Biologie als historische Wissenschaft Aussagen über Ereignisse oder Zustände in der Vergangenheit (retrodiction, Oh 2010). Über Fossilfunde werden mit Hilfe abduktiven Schließens beispielsweise Erklärungen über die Vergangenheit geologischer Ereignisse rekonstruiert (Oh 2019). In seinen didaktischen Forschungsarbeiten stellt Oh (2010, 2019) eine enge Verbindung zwischen Abduktion und Modellieren her (modeling-based abductive reasoning, Oh 2019). Dabei sollen Lehramtsstudierende zu geologischen Phänomenen (evidence, Oh 2019) unter Rückgriff auf ihre konzeptionellen, epistemischen, emotionalen oder praktischen Ressourcen (resource model, Oh 2019), also auf Basis ihrer Vorstellungen, Überzeugungen, Einstellungen und praktischen Erfahrungen, ein Modell entwickeln, das das Phänomen bestmöglich erklärt (explanatory model, Clement 2008). Die Studien knüpfen an umfangreiche Untersuchungen von Clement (2008) bei physikalischen Problemstellungen zum abduktiven Schließen beim Modellieren an. In der Mathematikdidaktik sprechen Park und Lee (2018) von einer abductive nature of mathematical modeling und schreiben dem abduktiven Schließen beim mathematischen Modellieren eine zentrale Bedeutung zu. Die hier genannten Studien bringen dem abduktiven Schließen beim Modellieren große Aufmerksamkeit entgegen (vgl. Clement 2008), wobei der Fokus darauf liegt zu verfolgen, wie es gelingt, ein fachlich angemessenes Modell herzustellen. Der Übergang von der Herstellung in die Anwendung mit deduktiv abgeleiteten Hypothesen wird nicht weitergehend in den Blick genommen.

In der Biologiedidaktik zeigen Arbeiten zum Modellieren an verschiedenen Blackbox-Szenarien (Koch et al. 2015; Großmann 2019; Krell und Hergert 2019; Göhner und Krell 2020), dass abduktives Schließen gelingen kann, wenn ein theoretischer Hintergrund oder wenn Kreativität zur Interpretation von Daten zur Verfügung stehen. Dies kann zu einem wiederholten Wechsel zwischen abduktiven und deduktiven Schlüssen führen. Gelingt das Entwickeln einer Erklärung des inneren Mechanismus der Blackbox nicht zufriedenstellend (Göhner und Krell 2020), weil kein theoretisches Wissen zur Verfügung steht, Analogien nicht gefunden werden oder kreative Lösungen fehlen (Göhner und Krell 2018), zeigen sich auch keine deduktiven Schlüsse in einer Anwendung (Göhner und Krell 2020, 2021).

\section{Empirische Ausblicke}

In weiterführenden fachdidaktischen Untersuchungen wird sich zeigen, inwieweit der vollständige Modellierprozess, der in der Anwendung deduktives Ableiten und Prüfen von Hypothesen erfordert, vom abduktiven Schließen in der Herstellung abhängt. Dies zieht die Frage nach sich, ob aus verfügbarem Wissen oder Kreativität eine beste Erklärung für ein Phänomen herangezogen werden kann. Die im Beitrag vorgenommene Integration abduktiven Schließens in den Prozess des Modellierens lässt sich im Sinne eines abductive turn (Reichertz 2013) auf andere Arbeitsweisen ausweiten (vgl. Upmeier zu Belzen und Krüger 2019a), die im Unterricht monistisch als ein hypothetisch-deduktiver Erkenntnisweg betrachtet werden (Langlet 2016; Popper 2005) - mit Auswirkungen auf Nature of Science-Perspektiven (vgl. Kind und Osborne 2017; Heering und Kremer 2018). Es wird darüber nachzudenken sein, inwiefern Abduktion als Motor für neue Erkenntnisse gegenüber Induktion und Deduktion im hypothetisch-deduktiven Denkschema unterrepräsentiert ist und die Fokussierung auf das deduktive Ableiten von Hypothesen beim Experimentieren (Mayer 2007; Gut-Glanzmann und Mayer 2018) aus fachdidaktischer Sicht ohne das abduktive Schließen zu kurz greift.

So wie vor elf Jahren der Strukturvorschlag der Modellkompetenz eine Vielzahl empirischer Untersuchungen in der Grundlagenforschung (Krell et al. 2016) und für die Praxis (Upmeier zu Belzen und Krüger 2019b; Fleige et al. 2012) angestoßen hat, so stößt dieser theoretische Beitrag unmittelbar eine Reihe weiterer Untersuchungen an. Im Sinne von Grundlagenforschung ist zu klären, ob Kompetenzen abduktiven Schließens beim Modellieren einen Beitrag zur Theoriebildung im Feld der Erkenntnisgewinnung liefern und inwieweit für erfolgreiches Modellieren eines Phänomens theoretische Fachkenntnisse sowie kreatives Denken zum entsprechenden Kontext bedeutsam sind. Unter der Perspektive Kompetenzerfassung ist zu untersuchen, inwieweit biologische Problemstellungen zum Modellieren Potenziale bzw. Limitationen für einen abduktiv gestützten Herstellungsprozess besitzen. Schließlich ist unter dem Aspekt einer Kompetenzförderung zu klären, inwieweit verschiedene biologische Problemstellungen abduktives Schließen ermöglichen und damit die Entwicklung von Modellierkompetenz fördern. 
Zukünftig sollen empirische Arbeiten zu diesen Bereichen ausgehend von dem Kompetenzmodell der Modellierkompetenz durchgeführt werden. Dabei wird es notwendig sein, einerseits das Niveau IIIA zu operationalisieren und anschließend die Trennbarkeit der Niveaus II, IIIA und IIIB empirisch zu untersuchen. Befunde aus den geplanten Arbeiten sind sowohl für Forschung als auch Lehre bedeutsam, weshalb wir mit Carlson und Daehler (2019) schlieBen: ,Now, it is time to test the model.“

Funding Open Access funding enabled and organized by Projekt DEAL.

Open Access Dieser Artikel wird unter der Creative Commons Namensnennung 4.0 International Lizenz veröffentlicht, welche die Nutzung, Vervielfältigung, Bearbeitung, Verbreitung und Wiedergabe in jeglichem Medium und Format erlaubt, sofern Sie den/die ursprünglichen Autor(en) und die Quelle ordnungsgemäß nennen, einen Link zur Creative Commons Lizenz beifügen und angeben, ob Änderungen vorgenommen wurden.

Die in diesem Artikel enthaltenen Bilder und sonstiges Drittmaterial unterliegen ebenfalls der genannten Creative Commons Lizenz, sofern sich aus der Abbildungslegende nichts anderes ergibt. Sofern das betreffende Material nicht unter der genannten Creative Commons Lizenz steht und die betreffende Handlung nicht nach gesetzlichen Vorschriften erlaubt ist, ist für die oben aufgeführten Weiterverwendungen des Materials die Einwilligung des jeweiligen Rechteinhabers einzuholen.

Weitere Details zur Lizenz entnehmen Sie bitte der Lizenzinformation auf http://creativecommons.org/licenses/by/4.0/deed.de.

\section{Literatur}

Aduriz-Bravo, A. (2019). Semantic views on models: an appraisal for science education. In A. Upmeier zu Belzen, D. Krüger \& J. van Driel (Hrsg.), Towards a competence-based view on models and modeling in science education (S. 21-37). Springer.

Baalmann, W., \& Kattmann, U. (2000). Birkenspanner: Genetik im Kontext von Evolution. Unterricht Biologie, 24(260), 32-35.

Bailer-Jones, D. (1999). Tracing the development of models in the philosophy of science. In L. Magnani, N. Nersessian \& P. Thagard (Hrsg.), Model-based reasoning in scientific discovery (S. 23-40). New York: Kluwer.

Carlson, J., \& Daehler, K. R. (2019). The refined consensus model of pedagogical content knowledge in science education. In A. Hume, R. Cooper \& A. Borowski (Hrsg.), Repositioning pedagogical content knowledge in teachers' knowledge for teaching science (S. 77-94). Singapore: Springer.

Chiu, M.-H., \& Lin, J.-W. (2019). Modeling competence in science education. Disciplinary and Interdisciplinary Science Education Research, 1, 1-11.

Clement, J. (2008). Creative model construction in scientists and students. Dordrecht: Springer.

Clement, J., \& Núñez-Oviedo, M. C. (2003). Abduction and analogy in scientific model construction. Proceedings of NARST., Philadelphia. (S. 1-17).

Duden (2020). Erklären. https://www.duden.de/rechtschreibung/erkla eren. Zugegriffen: 11. Febr. 2021.

Fleige, J., Seegers, A., Upmeier zu Belzen, A., \& Krüger, D. (2012). Modellkompetenz im Biologieunterricht 7-10. Verlag: Auer.

Giere, R., Bickle, J., \& Mauldin, R. (2006). Understanding scientific reasoning. London: Thomson.

Godfrey-Smith, P. (2006). The strategy of model-based science. Biology \& Philosophy, 21, 725-740.
Göhner, M., \& Krell, M. (2018). Modellierungsprozesse von Lehramtsstudierenden der Biologie. Erkenntnisweg Biologiedidaktik, $17,45-61$.

Göhner, M., \& Krell, M. (2020). Preservice science teachers' strategies in scientific reasoning: the case of modeling. Research in Science Education. https://doi.org/10.1007/s11165-020-09945-7.

Göhner, M., \& Krell, M. (2021). Was ist schwierig am Modellieren? Identifikation und Beschreibung von Hindernissen in Modellierungsprozessen von Lehramtsstudierenden naturwissenschaftlicher Fächer. ZfDN. https://doi.org/10.1007/s40573-021-00131-4

Gouvea, J., \& Passmore, C. (2017). "Models of" versus "Models for". Science \& Education, 26, 49-63.

Grant, R., \& Weiner, J. (1999). Ecology and evolution of darwin's finches. : Princeton University Press.

Greve, W., \& Wentura, D. (1997). Wissenschaftliche Beobachtung. Eine Einführung. Weinheim: Beltz.

Großmann, L. (2019). Modellverstehen und Modellieren an einer Blackbox. Eine Videoanalyse aus der biologiedidaktischen Forschung. Wiesbaden: Springer Spektrum.

Gut-Glanzmann, C., \& Mayer, J. (2018). Experimentelle Kompetenz. In D. Krüger, I. Parchmann \& H. Schecker (Hrsg.), Theorien in der naturwissenschaftsdidaktischen Forschung (S. 121-140). Berlin: Springer.

Habermans, J. (1968). Erkenntnis und Interesse (S. 139-172). Frankfurt am Main: Suhrkamp.

Harman, G. (1965). The inference to the best explanation. The Philosophical Review, 74(1), 88-95.

Harré, R. (1970). The principles of scientific thinking. London, Basingstoke: Macmillan.

Heering, P., \& Kremer, K. (2018). Nature of science. In D. Krüger, I. Parchmann \& H. Schecker (Hrsg.), Theorien in der naturwissenschaftsdidaktischen Forschung (S. 105-119). Berlin: Springer.

Hodson, D. (2014). Learning science, learning about science, doing science: Different goals demand different learning methods. International Journal of Science Education, 36(15), 2534-2553.

Johnson, T., \& Krems, J. (2001). Use of current explanations in multicausal abductive reasoning. Cognitive Science, 25, 903-939.

Johnson, T.R., Krems, J., \& Amra, N. K. (1994). A computational model of human abductive skill and its acquisition. In A. Ram \& K. Eiselt (Hrsg.), Proceedings of the Sixteenth Annual Conference of the Cognitive Science Society (S. 463-468). Hillsdale: Erlbaum.

Kampourakis, K., \& Niebert, K. (2018). Explanation in biology education. In K. Kampourakis \& M. Reiss (Hrsg.), Teaching biology in schools. Global research, issues, and trends (S. 236-248). New York: Routledge.

Ke, L., \& Schwarz, C. (2019). Using epistemic considerations in teaching: fostering students' meaningful engagement in scientific modeling. In A. Upmeier zu Belzen, D. Krüger \& J. van Driel (Hrsg.), Towards a competence-based view on models and modeling in science education (S. 181-199). Cham: Springer.

Kind, P., \& Osborne, J. (2017). Styles of scientific reasoning: a cultural rationale for science education? Science Education, 101(1), 8-31.

Knuuttila, T. (2011). Modelling and representing: an artefactual approach to model-based representation. Studies in History and Philosophy of Science, 42, 262-271.

Koch, S., Krell, M., \& Krüger, D. (2015). Förderung von Modellkompetenz durch den Einsatz einer Blackbox. Erkenntnisweg Biologiedidaktik, 14, 93-108.

Krell, M., \& Hergert, S. (2019). The black box approach: analyzing modeling strategies. In A. Upmeier zu Belzen, D. Krüger \& J. van Driel (Hrsg.), Towards as competence-based view on models and modeling in science education (S. 147-160). Cham: Springer.

Krell, M., Upmeier zu Belzen, A., \& Krüger, D. (2016). Modellkompetenz im Biologieunterricht. In A. Sandmann \& P. Schmiemann (Hrsg.), Biologiedidaktische Forschung: Schwerpunkte und Forschungsstände (Bd. 1, S. 83-102). : Logos. 
Krell, M., Walzer, C., Hergert, S., \& Krüger, D. (2019). Development and application of a category system to describe pre-service science teachers' activities in the process of scientific modelling. Research in Science Education, 49(5), 1319-1345.

Krems, J., \& Johnson, T. (1995). Integration of anomalous data in multicausal explanations. In J. D. Moore \& J.F. Lehman (Hrsg.), Proceedings of the 1995 annual conference of the cognitive science society (S. 277-282). Hillsdale: Erlbaum.

Krems, J., Johnson, T., \& Kliegl, R. (1997). Kognitive Komplexität und abduktives Schließen. In R. H. Kluwe (Hrsg.), Strukturen und Prozesse intelligenter Systeme (S. 215-232). Wiesbaden: Deutscher Universitätsverlag.

Krüger, D., \& Krell, M. (2020). Maschinelles Lernen mit Aussagen zur Modellkompetenz. Zeitschrift für Didaktik der Naturwissenschaften, 36, 157-172.

Krüger, D., Kauertz, A., \& zu Belzen, U.A. (2018). Modelle und das Modellieren in den Naturwissenschaften. In D. Krüger, I. Parchmann \& H. Schecker (Hrsg.), Theorien in der naturwissenschaftsdidaktischen Forschung (S. 141-157). Berlin Heidelberg: Springer.

Langlet, J. (2016). Kultur der Naturwissenschaften. In H. GropengieBer, U. Harms \& U. Kattmann (Hrsg.), Fachdidaktik Biologie (S. 80-97). Hallbergmoos: Aulis.

Magnani, L. (1999). Model-based creative abduction. In L. Magnani, N. Nersessian \& P. Thagard (Hrsg.), Model-based reasoning in scientific discovery (S. 219-238). New York,: Kluwer Academic.

Magnani, L. (2004). Model-based and manipulative abduction in science. Foundations of Science, 9(3), 219-247.

Mahootian, F., \& Eastman, T.E. (2009). Complementary frameworks of scientific inquiry: hypothetico-deductive, hypothetico-inductive, and observational-inductive. World Futures, 65(1), 61-75.

Mahr, B. (2008). Ein Modell des Modellseins. In U. Dirks \& E. Knobloch (Hrsg.), Modelle (S. 187-218). Frankfurt am Main: Peter Lang.

Mahr, B. (2009). Die Informatik und die Logik der Modelle. Informatik-Spektrum, 32, 228-249.

Mahr, B. (2015). Modelle und ihre Befragbarkeit. Grundlagen einer allgemeinen Modelltheorie. Erwägen Wissen Ethik, 26(3), 329-342.

Mayer, J. (2007). Erkenntnisgewinnung als wissenschaftliches Problemlösen. In D. Krüger \& H. Vogt (Hrsg.), Theorien in der biologiedidaktischen Forschung (S. 177-186). Berlin: Springer.

Meyer, M. (2009). Abduktion, Induktion - Konfusion. Bemerkungen zur Logik der interpretativen Sozialforschung. Zeitschrift für Erziehungswissenschaften, 12(2), 302-320.

Mittelstraß, J. (2005). Anmerkungen zum Modellbegriff. In der Präsident, der Berlin-Brandenburgischen Akademie \& Wissenschaften (Hrsg.), Modelle des Denkens: Streitgespräch in der Wissenschaftlichen Sitzung der Versammlung der Berlin-Brandenburgischen Akademie der Wissenschaften am 12. Dezember (S. 65-67). Berlin: Berlin-Brandenburgische Akademie der Wissenschaften.

Morrison, M., \& Morgan, M. S. (1999). Introduction. In M. S. Morgan \& M. Morrison (Hrsg.), Models as mediators. Perspectives on natural and social science (S. 1-9). Cambridge: Cambridge University Press.

Nicolaou, C., \& Constantinou, C. (2014). Assessment of the modeling competence. Educational Research Review, 13, 52-73.

Oh, P.S. (2010). How can teachers help students formulate scientific hypotheses? Some strategies found in abductive inquiry activities of earth science. International Journal of Science Education, 32(4), 541-560.

Oh, P.S. (2011). Characteristics of abductive inquiry in earth science: an undergraduate case study. Science Education, 95, 409-430.
Oh, P.S. (2019). Features of modeling-based abductive reasoning as a disciplinary practice of inquiry in earth science. Science \& Education, 28, 731-757.

Park, J.H., \& Lee, K.-H. (2018). How can mathematical modeling facilitate mathematical inquiries? Focusing on the abductive nature of modeling. EURASIA Journal of Mathematics, Science and Technology Education. https://doi.org/10.29333/ejmste/92557.

Passmore, C., Gouvea, J., \& Giere, R. (2014). Models in science and in learning science. In M. Matthews (Hrsg.), International handbook of research in history, philosophy and science teaching (S. 1171-1202). Dordrecht: Springer.

Peirce, C. S. (1978). [Harvard] lectures on pragmatism. Collected Papers of C.S. Peirce, Bd. 5 (S. 171-172). Cambridge: Belknap, Harvard.

Popper, K. (2005). Logik der Forschung. Tübingen: Mohr Siebeck.

Reichertz, J. (2013). Die Abduktion in der qualitativen Sozialforschung (2. Aufl.). Berlin Heidelberg: Springer.

Rocksén, M. (2016). The many roles of "explanation" in science education: a case study. Cultural Studies of Science Education, 11, $837-868$.

Schurz, G. (2008). Patterns of abduction. Synthese, 164, 201-234.

Schwarz, C. V., Reiser, B. J., Davis, E. A., Kenyon, L., Achér, A., Fortus, D., Shwartz, Y., Hug, B., \& Krajcik, J. (2009). Developing a leaming progression for scientific modeling: making scientific modeling accessible and meaningful for learners. Journal of Research in Science Teaching, 46(6), 632-654.

Sekretariat der Ständigen Konferenz der Kultusminister der Länder in der Bundesrepublik Deutschland (2020). Bildungsstandards im Fach Biologie für die Allgemeine Hochschulreife. Bonn: Berlin.

Shemwell, J. T., \& Capps, D.K. (2019). Using epistemic considerations in teaching: fostering students' meaningful engagement in scientific modeling. In A. Upmeier zu Belzen, D. Krüger \& J. van Driel (Hrsg.), Towards a competence-based view on models and modeling in science education (S. 291-307). Cham: Springer.

Sturm, G. (2006). Abduktion. In J. Behnke, T. Gschwend, D. Schindler \& K.-U. Schnapp (Hrsg.), Methoden der Politikwissenschaft. Neuere qualitative und quantitative Analyseverfahren (S. 27-35). Baden-Baden: Nomos.

Trommler, F., \& Hammann, M. (2020). The relationship between biological function and teleology: implications for biology education. Evo Edu Outreach. https://doi.org/10.1186/s12052-02000122-y.

Upmeier zu Belzen, A., \& Krüger, D. (2010). Modellkompetenz im Biologieunterricht. Zeitschrift für Didaktik der Naturwissenschaften, 15, 41-57.

Upmeier zu Belzen, A., \& Krüger, D. (2019a). Modelle und Modellieren im Biologieunterricht: Ein Fall für Erkenntnisgewinnung. Unterricht Chemie, 171, 38-41.

Upmeier zu Belzen, A., \& Krüger, D. (2019b). Modelle als methodische Werkzeuge begreifen und nutzen: Empirische Befunde und Empfehlungen für die Praxis. In J. Groß, M. Hammann, P. Schmiemann \& J. Zabel (Hrsg.), Biologiedidaktische Forschung: Erträge für die Praxis. Berlin Heidelberg: Springer.

Upmeier zu Belzen, A., van Driel, J., \& Krüger, D. (2019). Introducing a framework for modeling competence. In A. Upmeier zu Belzen, D. Krüger \& J. van Driel (Hrsg.), Towards as competence-based view on models and modeling in science education (S. 3-19). Berlin Heidelberg: Springer.

Wirth, U. (2003). Die Phantasie des Neuen als Abduktion. Deutsche Vierteljahrsschrift für Literaturwissenschaft und Geistesgeschichte, 77(4), 591-618. 